\title{
Mean flow effects in the nearly inviscid Faraday waves
}

\author{
E. Martín ${ }^{1} \&$ J. M. Vega ${ }^{2}$ \\ ${ }^{1}$ E. T. S. Ingenieros Industriales, Universidad de Vigo, Spain \\ ${ }^{2}$ E. T. S. Ingenieros Aeronáuticos, \\ Universidad Politécnica de Madrid, Spain
}

\begin{abstract}
We study the weakly nonlinear evolution of Faraday waves in a two dimensional version of a vertically vibrating annular container. In the small viscosity limit, the evolution of the surface waves is coupled to a non-oscillatory mean flow that develops in the bulk of the container. A system of equations is derived for the coupled slow evolution of the spatial phase of the surface wave and the streaming flow. These equations are numerically integrated to show that the simplest reflection symmetric steady state (the usual array of counter-rotating eddies below the surface wave) becomes unstable for realistic values of the parameters. The new states include limit cycles, steadily travelling waves (which are standing in a moving reference frame), and some more complex attractors. We also consider the effect of surface contamination, modelled by Marangoni elasticity with insoluble surfactant, in promoting drift instabilities in spatially uniform standing Faraday waves. It is seen that contamination enhances drift instabilities that lead to various steadily propagating and (both standing and propagating) oscillatory patterns. In particular, steadily propagating waves appear to be quite robust, as in the experiment by Douady et al. (1989).

Keywords: Faraday instability, mean flow, weakly nonlinear analysis, Marangoni elasticity.
\end{abstract}

\section{Introduction}

We consider the parametric excitation of waves at the free surface of a horizontal liquid layer that is being vertically vibrated. If the forcing amplitude exceeds a threshold value, the system exhibits surface waves that are named after 
Faraday [1]. These waves have attracted a great deal of attention, especially because of the rich variety of non-linear pattern forming phenomena promoted by the Faraday instability [2, 3, 4]. Unfortunately, current theoretical approaches fail to appropriately explain essential issues associated with the behavior beyond threshold, particularly in the singular limit of small viscosity. The usual nonlinear amplitude equations used to describe this weakly nonlinear regime are obtained from a strictly inviscid formulation and corrected a posteriori by adding some linear dissipation terms $[2,3]$. This formulation ignores the presence of the slow non oscillatory mean flow that is driven by the boundary layers at the container walls and free surface and, in the case of a monochromatic wave only predicts standing waves (SW) after onset and fails to reproduce the drifting SWs that have been observed experimentally in annular containers [5, 6]. The object of the present paper is precisely to analyze the coupled evolution of the surface waves and the mean flow.

The remaining of the paper is organized as follows: in $\S 2$ we shall present the systems of equations for the slow time evolution of the surface waves and the mean flow, derived from a exact formulation based on the full Navier-Stokes equations. This will be done assuming either a clean free surface and a contaminated one (surface contamination is likely to be present in water, as in [5], unless care is taken in the experimental set-up). The relevant patterns obtained for large-time resulting from the primary bifurcation will be described and discussed in $\S 3$, where some conclusions will also be made.

\section{Coupled amplitude-mean flow equations}

We consider a horizontal 2-D liquid layer supported by a vertically vibrating plate (fig.1), and use the container's depth $h$ and the gravitational time $\sqrt{h / g}$ for nondimensionalization. The governing equations are the following

$$
\begin{aligned}
& u_{x}+v_{y}=0, \\
& u_{t}+v\left(u_{y}-v_{x}\right)=-q_{x}+C\left(u_{x x}+u_{y y}\right), \\
& v_{t}-u\left(u_{y}-v_{x}\right)=-q_{y}+C\left(v_{x x}+v_{y y}\right), \\
& u=v=0 \quad \text { at } \quad y=-1, \\
& v=f_{t}+u f_{x}, \quad C^{1 / 2}\left(\hat{u}_{n}+\hat{v}_{s}+\kappa \hat{u}\right)=0, \\
& \quad q-\frac{u^{2}+v^{2}}{2}+4 \omega^{2} \varepsilon f \cos (2 \omega t)-f+T \kappa=2 C \hat{v}_{n} \quad \text { at } y=f, \\
& u, v, q \text { and } f \text { are } L \text {-periodic in } x,
\end{aligned}
$$

where

$$
s=\int_{0}^{x} \sqrt{1+f_{x}^{2}} d x \text { and } \kappa=\frac{f_{x x}}{\left(1+f_{x}^{2}\right)^{3 / 2}}
$$

are an arch length parameter and the curvature of the free surface (defined as $y=f$ ), respectively, and $n$ is a coordinate along the upward unit normal to the 


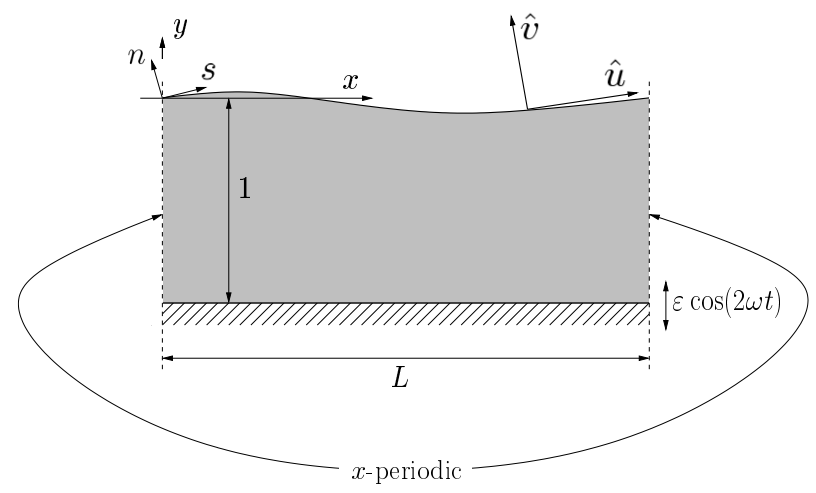

Figure 1: Sketch of the fluid domain.

free surface; $\hat{u}$ and $\hat{v}$ are the tangential and normal velocity components at the free surface $y=f$, which are related to the horizontal and vertical components $u$ and $v$ by

$$
\hat{u}=\frac{u+f_{x} v}{\sqrt{1+f_{x}^{2}}}, \quad \hat{v}=\frac{v-f_{x} u}{\sqrt{1+f_{x}^{2}}} .
$$

Equations (1)-(6) formulate the problem assuming a clean free surface, with no contamination. Nevertheless, surface contamination is likely to be present in water. The only difference between the clean and contaminated cases is seen in the boundary condition (5b), whose right hand side was zero for the clean surface and now accounts for the presence of contaminating surfactants (9), modelled in the simplest way: the resulting tangential stress includes Marangoni elasticity effects produced by a variation of surface tension with surfactant concentration

$$
C^{1 / 2}\left(\hat{u}_{n}+\hat{v}_{s}+\kappa \hat{u}\right)=-\gamma \zeta_{s} .
$$

A linear law is assumed for the variation of the surface tension $T^{*}$ with the surfactant concentration $\zeta^{*}$, namely $T^{*}\left(\zeta^{*}\right)=T_{0}^{*}+\left(d T^{*} / d \zeta_{0}^{*}\right)\left(\zeta^{*}-\zeta_{0}^{*}\right)$, where the derivative is calculated at the equilibrium value of the surfactant concentration $\zeta^{*}$, denoted as $\zeta_{0}^{*}$.

The nondimensional surfactant concentration $\zeta=\left(\zeta^{*}-\zeta_{0}^{*}\right) / \zeta_{0}^{*}$ is given by the conservation equation for an insoluble surfactant

$$
\zeta_{t}+[(1+\zeta) u]_{s}=0 \quad \text { in } 0<s<s_{L}, \quad \zeta\left(s+s_{L}, t\right)=\zeta(s, t) .
$$

Here, $s_{L}$ is the length of the free surface in one period and we are neglecting both cubic terms and surface diffusion of the surfactant.

Both dimensionless problems, namely (1)-(6) and (1)-(5a),(5c)-(6), (9), (10), depend on the following nondimensional parameters: the forcing frequency $2 \omega=$ $2 \omega^{*} \sqrt{h / g}$ and amplitude $\varepsilon=\varepsilon^{*} / h$, the ratio of viscous to gravitational effects 
$C=\mu /\left(\rho \sqrt{g h^{3}}\right)(\rho=$ density, $\mu=$ viscosity $)$, the Bond number $T^{-1}=\rho g h^{2} / T_{0}^{*}$ ( $T_{0}^{*}=$ surface tension at equilibrium), the horizontal aspect ratio $L=L^{*} / h$ ( $L^{*}=$ horizontal length of the domain), and only for the contaminated free surface problem, the Marangoni elasticity number $\gamma=\zeta_{0}^{*}\left(d T^{*} / d \zeta_{0}^{*}\right) C^{1 / 2} /(\mu \sqrt{g h})$.

We shall consider small, nearly-resonant solutions at small viscosity and conveniently rescaled Marangoni elasticity, i.e.,

$$
|u|+|v|+|q|+|f|+|\zeta| \ll 1, \quad \varepsilon \ll 1, \quad\left|\omega-\omega_{0}\right| \ll 1, \quad C \ll 1, \quad \gamma \sim 1,
$$

where $\omega_{0}$ is a natural frequency in the inviscid limit $(C=0)$. The assumption that $C \ll 1$ is reasonable for not too viscous fluids in not too thin layers. The assumption that $\gamma \sim 1$ is made for the Marangoni elasticity to have a significant effect both in the damping ratio of the surface waves and in the streaming flow (see [8] for more details). As explained in [7] and [9], the solution can be expanded distinguishing between an oscillating part caused by the oscillatory inviscid modes (with a $\mathcal{O}(1)$ frequency and a $\mathcal{O}(\sqrt{C})$ decay rate) and a slow non-oscillatory part generated by the viscous modes (with a $\mathcal{O}(C)$ decay rate), which produce the mean flow, denoted hereinafter by the superscript $m$. The solution in the bulk region, outside the boundary layers that appear at the free surface and the bottom plate, is written as follows

$$
\begin{aligned}
& u=U_{0}(y) e^{i \omega t}\left[A(t) e^{i k x}-B(t) e^{-i k x}\right]+c . c .+u^{m}(x, y, t)+\cdots, \\
& v=i V_{0}(y) e^{i \omega t}\left[A(t) e^{i k x}+B(t) e^{-i k x}\right]+c . c .+v^{m}(x, y, t)+\cdots, \\
& q=Q_{0}(y) e^{i \omega t}\left[A(t) e^{i k x}+B(t) e^{-i k x}\right]+c . c .+q^{m}(x, y, t)+\cdots, \\
& f=e^{i \omega t}\left[A(t) e^{i k x}+B(t) e^{-i k x}\right]+\text { c.c. }+f^{m}(x, t)+\cdots, \\
& \zeta=\Xi_{0} e^{i \omega t}\left[A(t) e^{i k x}+B(t) e^{-i k x}\right]+c . c .+\zeta^{m}(x, t)+\cdots,
\end{aligned}
$$

where $c . c$ stands for the complex conjugate, $k=2 m \pi / L$ (with $m$ a positive integer) is the horizontal wave number and $U_{0}, V_{0}$ and $Q_{0}$ are the corresponding inviscid eigenfunctions

$$
\begin{aligned}
& U_{0}=-\frac{k Q_{0}}{\omega_{0}}, \quad V_{0}=\frac{Q_{0 y}}{\omega_{0}}, \quad Q_{0}=\frac{\omega_{0}^{2} \cosh k(y+1)}{k \sinh k}, \\
& \omega_{0}^{2}=k\left(1+T k^{2}\right) \tanh k .
\end{aligned}
$$

Note that the expansion for the surfactant concentration variable (12e) is only necessary for the contaminated problem, where

$$
\Xi_{0}=\left(k \omega_{0} \sqrt{i \omega_{0}}\right) /\left(\tanh k\left(\omega_{0} \sqrt{i \omega_{0}}-i k^{2} \gamma\right)\right)
$$

cannot be obtained in the inviscid approximation. Dependence of the complex amplitudes $A$ and $B$ on $x$ is ignored for simplicity, see [10] and [11] for a more complicated analysis including spatial wave modulations. The weakly nonlinear analysis requires the amplitudes $A$ and $B$ to be small and depend slowly on time $\left|A^{\prime}\right| \ll|A| \ll 1,\left|B^{\prime}\right| \ll|B| \ll 1$. 
If we insert expansions (12a)-(12d) into the governing equations for the clean free surface case, and (12a)-(12e) into the equations for the contaminated free surface problem, take into account the boundary layers at the free surface and the bottom of the container, and apply solvability conditions, the following equations for the evolution of the complex amplitudes are obtained

$$
\begin{aligned}
& A^{\prime}=\left[-d_{1}-i d_{2}+\mathrm{i} \alpha_{3}|A|^{2}-\mathrm{i} \alpha_{4}|B|^{2}-\mathrm{i} \frac{\alpha_{6}}{L} \int_{-1}^{0} \int_{0}^{L} g(y) u^{m} d x d y\right] A+\mathrm{i} \varepsilon \alpha_{5} \bar{B}, \\
& B^{\prime}=\left[-d_{1}-i d_{2}+\mathrm{i} \alpha_{3}|B|^{2}-\mathrm{i} \alpha_{4}|A|^{2}+\mathrm{i} \frac{\alpha_{6}}{L} \int_{-1}^{0} \int_{0}^{L} g(y) u^{m} d x d y\right] B+\mathrm{i} \varepsilon \alpha_{5} \bar{A},
\end{aligned}
$$

which depend on the mean flow through a non local term. See [7] and [8] for a detailed derivation of the equations above and for the expressions of the coefficients and the function $g(y)$ in the non contaminated and contaminated case, respectively.

The solution of equations (16) and (17) always relaxes to a standing wave $(|A|=$ $|B|=R_{0}$ ) of the form

$$
f(x, t)=4 R_{0} \cos \left(\omega t+\phi_{0}\right) \cos [k(x-\psi)]
$$

with constant amplitude $R_{0}$ (which depends on the amplitude of the applied forcing) and spatial phase $\psi(t)$, which remains coupled to the streaming flow through the equation

$$
\psi^{\prime}=\frac{\alpha_{6}}{k L} \int_{-1}^{0} \int_{0}^{L} g(y) u^{m} d x d y .
$$

Ignoring the initial transient, taking into account the last result in expansions (12a)(12e), and introducing these expressions in the two cases, we obtain the following equations for the mean flow outside the two boundary layers

$$
\begin{aligned}
& \tilde{u}_{x}+\tilde{v}_{y}=0, \\
& \frac{\partial \tilde{u}}{\partial \tau}+\tilde{v}\left(\tilde{u}_{y}-\tilde{v}_{x}\right)=-\tilde{q}_{x}+R e^{-1}\left(\tilde{u}_{x x}+\tilde{u}_{y y}\right), \\
& \frac{\partial \tilde{v}}{\partial \tau}-\tilde{u}\left(\tilde{u}_{y}-\tilde{v}_{x}\right)=-\tilde{q}_{y}+R e^{-1}\left(\tilde{v}_{x x}+\tilde{v}_{y y}\right), \\
& \tilde{u}, \tilde{v} \text { and } \tilde{q} \quad \text { are } x \text {-periodic, of period } L=2 m \pi / k, \\
& \frac{d \psi}{d \tau}=\frac{1}{L} \int_{-1}^{0} \int_{0}^{L} G(y) \tilde{u}(x, y, \tau) d x d y, \quad G(y)=\frac{2 k \cosh 2 k(y+1)}{\sinh 2 k}
\end{aligned}
$$

where the bottom and free surface horizontal velocities are determined from one of the following additional conditions, either

$$
\begin{aligned}
& \tilde{u}=-\sin [2 k(x-\psi)], \quad \tilde{v}=0 \quad \text { at } y=-1, \\
& \tilde{u}_{y}=0, \quad \tilde{v}=0, \quad \text { at } y=0,
\end{aligned}
$$




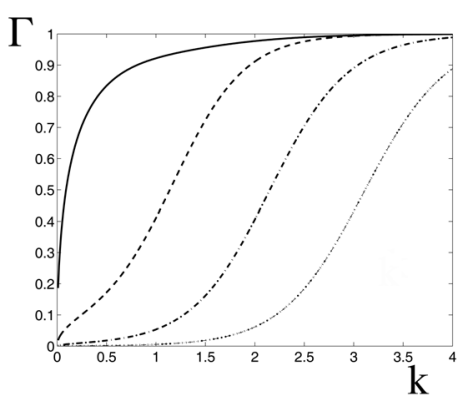

(a)

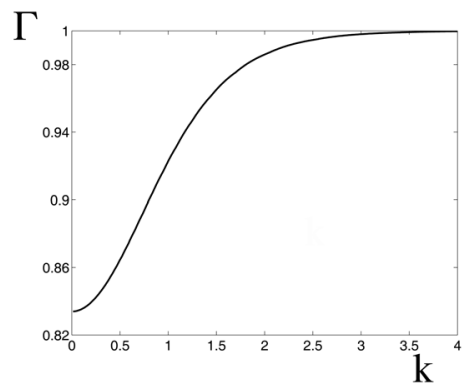

(b)

Figure 2: The contamination parameter $\Gamma$. (a) $\Gamma$ vs. $k$ for $T=7.42 \cdot 10^{-4}$ and: $(-) \gamma=1,(---) \gamma=0.1,(-\cdots-\cdot-) \gamma=0.01$, and $(\cdots \cdots)$ $\gamma=10^{-3}$. (b) The maximum value of $\Gamma$ vs. $k$ for $T=7.42 \cdot 10^{-4}$ and varying $\gamma$.

or the clean free surface case, or

$$
\begin{aligned}
& \tilde{u}=-(1-\Gamma) \sin [2 k(x-\psi)], \quad \tilde{v}=0 \quad \text { at } y=-1, \\
& \tilde{u}=-\Gamma \sin [2 k(x-\psi)]+\tilde{u}_{0}(\tau), \quad \tilde{v}=0, \quad \int_{0}^{L} \tilde{u}_{y} d x=0, \quad \text { at } y=0,
\end{aligned}
$$

for the contaminated free surface. For convenience, we have rescaled time and mean flow variables as

$$
\tau=\operatorname{ReCt}, \quad \tilde{u}=\frac{u^{m}}{\operatorname{ReC}}, \quad \tilde{v}=\frac{v^{m}}{\operatorname{ReC}}, \quad \tilde{q}=\frac{q^{m}}{(\operatorname{Re} C)^{2}},
$$

with the effective mean flow Reynolds number defined as follows

$$
R e=\frac{2 R_{0}^{2}}{C}\left(\alpha_{7}+\alpha_{8}\right)
$$

with

$$
\alpha_{7}=\frac{3 \omega_{0} k}{\sinh ^{2} k}, \quad \alpha_{8}=\frac{\omega_{0} k}{\tanh ^{2} k}\left(\frac{4 \gamma k^{2}}{\omega_{0} \sqrt{i \omega_{0}}-i \gamma k^{2}}+c . c+\frac{3 \gamma^{2} k^{4}}{\left|\omega_{0} \sqrt{i \omega_{0}}-i \gamma k^{2}\right|^{2}}\right) .
$$

where $\gamma$ must be substituted by 0 in (31) when considering the clean surface case. Equations for the clean case (20)-(26), hereinafter referred to as MFClean, depend on the values of the 3 parameters $(R e, k, m)$, while the contaminated free surface problem defined by (20)-(24), (27)-(28), hereinafter MFContam, depends on an 
additional contamination parameter $\Gamma$ that measures the relative effect of contamination in the generation of the streaming flow

$$
\Gamma=\Gamma(k, T, \gamma) \equiv \frac{\alpha_{8}}{\alpha_{7}+\alpha_{8}},
$$

and is plotted $v s$. the wavenumber $k$ in figure 2(a) for the indicated values of $\gamma$; the selected value of the inverse of the Bond number, $T=7.42 \cdot 10^{-4}$, corresponds to a $10 \mathrm{~cm}$ depth water container. It can be seen that for deep water problems, namely $k>\pi$, the contamination parameter is of the order of 1 , even for quite small values of the Marangoni number $\gamma$. The maximum values of $\Gamma$ are plotted in figure 2(b).

\section{Results and conclusions}

Problems MFClean and MFContam are both numerically solved to show that for small values of the effective mean flow Reynolds number $R e$, the solution relaxes to the basic standing wave (SW) with $\psi^{\prime}=0$. The mean flow associated with this basic SW consists of an array of pairs of steady counterrotating eddies. Examples are plotted in figure 3(a) for clean free surface and in fig.4(a)-4(c) for contaminated free surface. These steady solutions are $L / 2$-symmetric and since they are also reflection symmetric in $x$, the integral (24) vanishes and the streaming flow does not affect the surface SW.

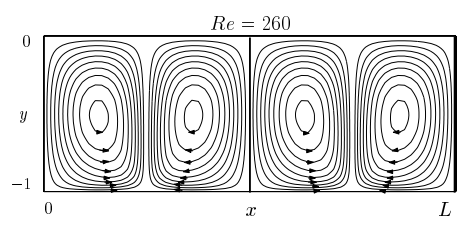

(a)

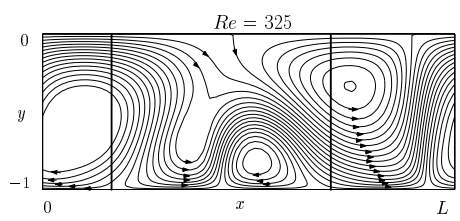

(b)

Figure 3: Streamlines of the streaming flow of MFClean for $k=2.37, L=2.65$ ( $m=1$ ) and (a) $R e=260$ and (b) $R e=325$ (in moving axes $x-\psi^{\prime} \tau$ with constant drift velocity). Thick vertical lines correspond to the nodes of the surface waves given by (18).

For the MFClean problem, if Re exceeds a threshold value, indicated in figure 5(a), the basic steady solution becomes unstable always through a Hopf bifurcation and a branch of time periodic solutions (PSW) appears, which produces a time periodic drift of the SW with no net drift on the free surface. These periodic solutions resemble locally the so called compression modes that have been observed in annular containers ([5], [6]) and cannot be obtained with the usual amplitude equations that ignore the coupling with the streaming flow. For some values of $k$ and $L$, there are some additional bifurcations to steadily traveling waves (TWs), which move at a constant speed like the one shown in figure 3(b), and more complex oscillatory attractors, but these depend strongly on $k$ and $L$ (see [7] 


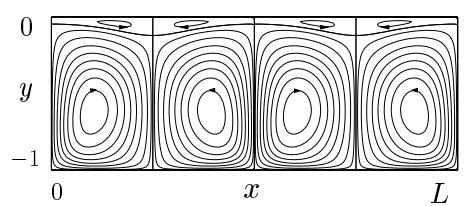

(a)

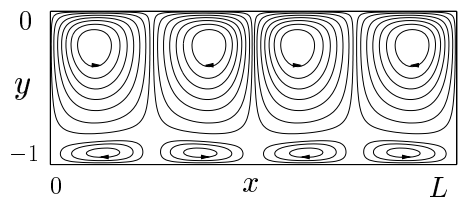

(c)

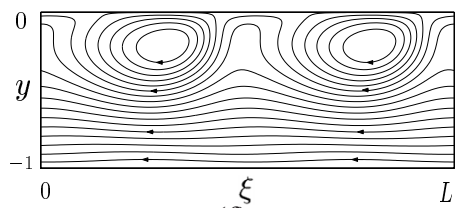

(f)

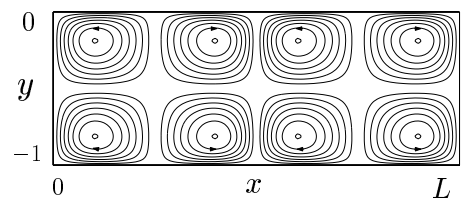

(b)

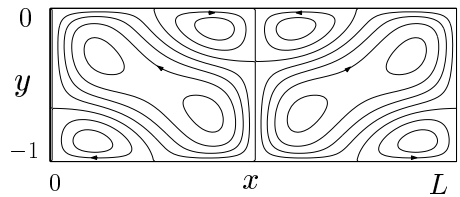

(d)

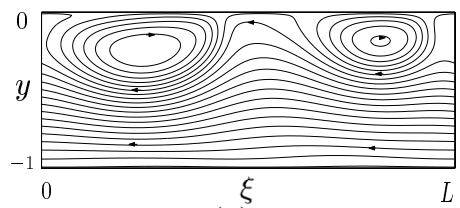

$(\mathrm{g})$

Figure 4: Streamlines of MFContam, for $k=2.37, L=2.65(m=1)$, and $(R e, \Gamma):$ (a) $(200,0.1)$, (b) $(160,0.5)$, (c) $(60,0.9)$, (d) $(200,0.5),(\mathrm{e})$ $(200,0.9)$ in moving axes $\xi=x-\psi^{\prime} \tau$ with constant drift velocity $\psi^{\prime}=$ 0.32 and $\tilde{u}_{0}=0.49$, (f) $(600,0.9)$ in moving axes with constant drift velocity $\psi^{\prime}=0.27$ and $\tilde{u_{0}}=0.53$.

for more details). However, drift instabilities were quite robust in the experiment of Douady et al. [5] (Fauve, personal communication, 2003) and the MFclean problem does not seem to reproduce this feature.

In order to mimic the behavior of tap water (used in the experiment [5]) the MFContam problem is solved to obtain that the primary instability of the basic $\mathrm{SW}(\mathrm{SW}(L / 2))$ depends on the value of the contamination parameter. In figure 5 (b) it can be seen that for small values of $\Gamma$ the instability takes place through a Hopf bifurcation and for quite small values of $\Gamma$ the contamination effect seems to stabilize the basic SWs (note that the critic Reynolds number for the clean case for the same values of $k$ and $L$ is marked with a large point in the horizontal axe of figure 5(b)).

This is because the only effect of contamination in this regime on the mean flow is to replace the free stress boundary condition at the free surface by a no-slip boundary condition, which reduces the strength of the mean flow. For an intermediate value of $\Gamma$ a symmetry breaking bifurcation to another type of SW no longer $L / 2$ symmetric (SW $(L)$ ) occurs, see figure 4(d) as an example. For larger values of the contamination parameter $\Gamma$ the basic $\operatorname{SW}(L / 2)$ destabilizes through a parity breaking bifurcation that leads to TWs $(\mathrm{TW}(L / 2))$ whose streamlines for the mean flow in a moving reference frame are similar to the one plotted in 4(e). 


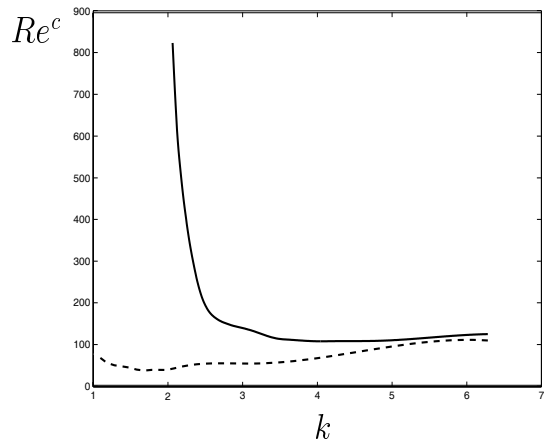

(a)

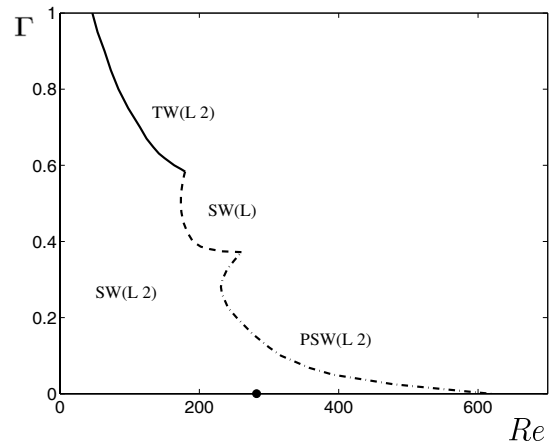

(b)

Figure 5: The primary instability of the basic SW for: (a) MFClean for different wave numbers $k$ and (b) MFContam, for $k=2.37, L=2.65(m=1)$. Figure 5(a): The bifurcation is always a Hopf bifurcation (- -$)((---)$ shows the parity breaking bifurcation that takes place only if the coupling between the surface wave and the mean flow (24) it is ignored). Figure 5(b): The bifurcation is either a Hopf bifurcation $(-\cdot-\cdot-)$ if $0<\Gamma<$ 0.372 , a $(L / 2)$-symmetry breaking bifurcation $(---)$ if $0.372<\Gamma<$ 0.584 , or a parity breaking bifurcation (-) if $0.584<\Gamma<1$.

Note that the mean flow is still $L / 2$-symmetric. In contrast with the clean case, these TWs appear in a primary bifurcation and are quite robust (remain unchanged for larger domains and appear for all values of the wave number we have checked). Thus, contamination effects seem to play an important role in the surface waves dynamics. For larger values of the Reynolds number $R e$, different secondary instabilities are obtained, which include another type of TWs with no $L / 2$-symmetric mean flow (figure 4(f)), pulsating traveling waves, and even chaotic attractors ([8]). For all these states that are not steady SW, the coupling with the mean flow is an essential ingredient that should not be ignored.

\section{Acknowledgements}

This work was supported by the National Aeronautics and Space Administration (Grant NNC04GA47G) and the Spanish Ministry of Education (Grant MTM200403808).

\section{References}

[1] Faraday, M. On the forms and states assumed by fluids in contact with vibrating elastic surfaces. Phil. Trans. R. Soc. Lond., 121, pp. 319-340, 1831.

[2] Miles, J. and Henderson D. On the forms and states assumed by fluids in contact with vibrating elastic surfaces. Annu. Rev. Fluid Mech., 22, pp. 143-165, 1990. 
[3] Cross, M. and Hohenberg, P.C. Pattern formation outside of equilibrium. Rev. Mod. Phys., 65, pp. 851-1112, 1993.

[4] Kudrolli, A. and Gollub, J.P. Patterns and spatio-temporal chaos in parametrically forced surface waves: A systematic survey at large aspect ratio. Physica D, 97, pp. 133-154, 1997.

[5] Douady, S. Fauve, S. and Thual, O., Oscillatory phase modulation of parametrically forced surface waves. Europhys. Lett., 10, pp. 309-315, 1989.

[6] Thual, O., Douady, S. and Fauve, S., Instabilities and Nonequilibrium Structures II. Ed. Tirapegui, E. and Villaroel, D., Kluwer, pp. 227, 1989

[7] Martín, E., Martel, C. and Vega, J.M. Drift instability of standing Faraday waves. J. Fluid Mech., 467, pp. 57-79, 2002.

[8] Martín, E. \& Vega, J.M. The effect of surface contamination on the drift instability of standing Faraday waves. J. Fluid Mech., in press (2005).

[9] Martel C. \& Knobloch, E., Damping of nearly-inviscid water waves. Phys. Rev. E, 56, pp. 5544-5548, 1997.

[10] Vega, J.M., Knobloch, E. and Martel, C., Nearly inviscid Faraday waves in annular containers of moderately large aspect ratio. Physica D,154, pp. 147171,2001

[11] Lapuerta, V., Martel, C. and Vega, J.M., Interaction of nearly-inviscid Faraday waves and mean flows in 2-D containers of quite large aspect ratio. Physica D, 173, pp. 178-203, 2002. 